\title{
Una aplicación móvil para el monitoreo de cultivos: caso de estudio campaña contra el pulgón amarillo del sorgo
}

\author{
Alejandra Perez-Mena ${ }^{1}$, José Alberto Fernández-Zepeda ${ }^{1}$, Juan Pablo Rivera-Caicedo², \\ Himer Avila-George ${ }^{3}$
}

perezmena@cicese.edu.mx, fernan@cicese.mx, jprivera@uan.edu.mx, himer.avila@ academicos.udg.mx

${ }^{1}$ Centro de Investigación Científica y de Educación Superior de Ensenada, Ensenada, 2286o, Baja California, México.

${ }^{2}$ CONACYT-UAN, Centro Nayarita de Innovación y Transferencia Tecnológica, Tepic, 63173, Nayarit, México.

${ }^{3}$ Centro Universitario de los Valles, Universidad de Guadalajara, Ameca, 466oo, Jalisco, México.

DOI: 10.17013/risti.31.118-133

Resumen: En este artículo, se introduce PulAm, una aplicación móvil basada en el sistema operativo móvil Android. Esta aplicación se diseñó como una herramienta de apoyo en el proceso de monitoreo de plagas de diferentes cultivos. Como caso de estudio, se introduce el monitoreo de cultivos de sorgo, en el estado de Nayarit, México; específicamente para la campaña contra el "pulgón amarillo" del sorgo.

Palabras-clave: aplicación móvil; sorgo; plaga del pulgón amarillo.

\section{A mobile application for crop monitoring: case study campaign against the yellow sorghum aphid}

Abstract: In this manuscript, PulAm is presented, a mobile application based on the Android mobile operating system. This application was designed as a support tool in the process of monitoring pests of different crops. As a case study, its use is introduced in the monitoring of sorghum crops in the state of Nayarit, Mexico; Specifically, for the campaign against the "yellow aphid" of sorghum.

Keywords: mobile app; sorghum; yellow aphid.

\section{Introducción}

La agricultura es una actividad primaria y de gran relevancia para el ser humano desde el punto de vista social, económico y ambiental. Lo anterior debido a que genera un gran número de trabajos y productos alimenticios; además, ayuda a conservar el suelo y la biodiversidad. 
El sorgo es el segundo cultivo más importante para la economía mexicana. La superficie destinada para la producción de sorgo es 1,720,208 hectáreas (SIAP, 2017). Según el Marco Censal Agropecuario, a excepción de la Ciudad de México, todos los estados del país tienen un porcentaje de superficie destinada al cultivo del sorgo (INEGI, 2016).

En la Figura 1 se puede apreciar un mapa de México donde se resalta en tonos naranja las zonas donde se cultiva el sorgo (el mapa se generó con la herramienta mapa digital de México (INEGI, 2019)), donde los estados de Tamaulipas y Sinaloa son los mayores productores de sorgo (SAGARPA, 2014).

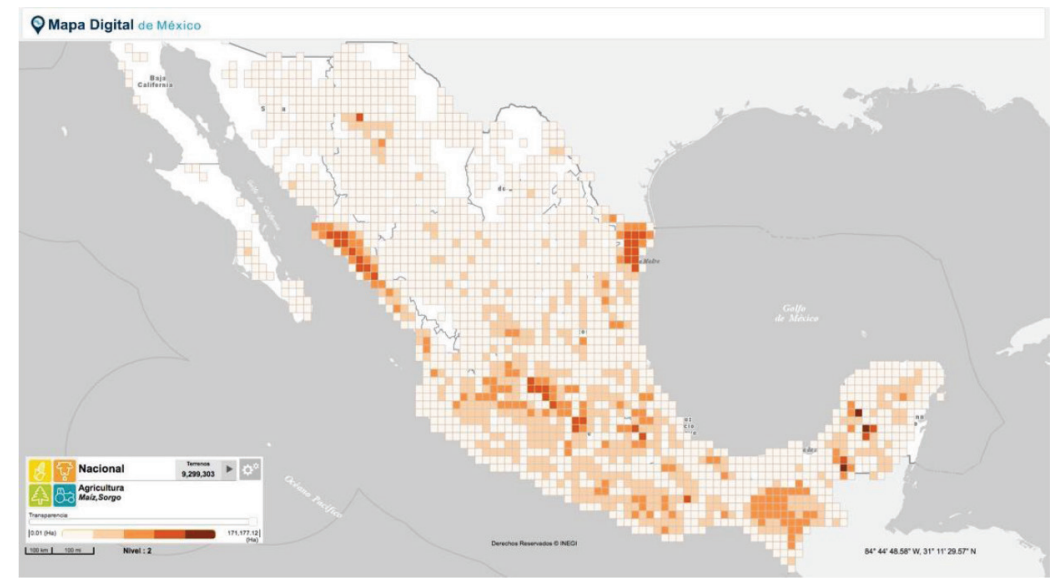

Figura 1 - Zonas de cultivos de sorgo en México

En el año 2013 apareció por primera vez en el territorio mexicano el virus del pulgón amarillo (Melanaphis sacchari), dañando severamente los cultivos de sorgo. Desde entonces, la producción de sorgo ha decrecido entre 30\% y 100\% en muchas áreas (Rodríguez-del-Bosque \& Terán, 2015). Esta plaga se ha establecido en prácticamente todo el territorio nacional debido a su alta capacidad de dispersión y elevado potencial de reproducción.

El pulgón amarillo es un insecto clasificado en la familia Aphididae, dicho insecto tiene hábitos gregarios, i.e., vive en colonias abundantes, específicamente en el lado posterior de las hojas de las plantas invadidas. Inicialmente, las colonias de pulgón amarillo aparecen en la base de la planta del sorgo y gradualmente se mueve a la parte alta, en donde pueden infestar la panícula del sorgo en floración (Rodríguez-del-Bosque \& Terán, 2015).

Una planta de sorgo con la plaga del pulgón amarillo muestra un cambio de coloración en la hoja, ya que este insecto se alimenta de la savia de la planta. Este proceso causa pérdida de nutrientes y estrés en la planta, ver Figura 2. El insecto también secreta una sustancia azucarada en la superficie de las hojas que propicia la aparición de moho y promueve la expansión de algunos virus como el Sugarcane mosaic (ScMV) o Sugarcane yellow leaf $(S c Y L V)$. Sin embargo, el daño más crítico en el cultivo es la reducción de la calidad del cultivo y del rendimiento de la cosecha (SENASICA, 2014). 


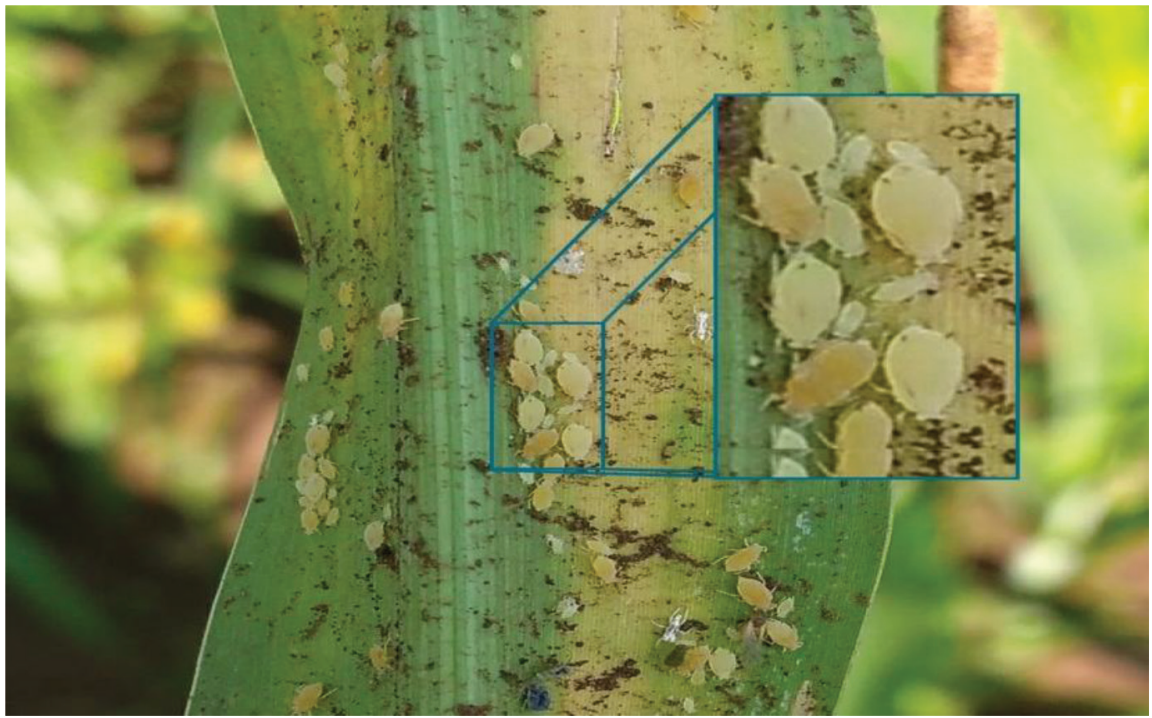

Figura 2 - Hojas de sorgo infestadas por pulgón amarillo

Dada la importancia económica del cultivo del sorgo en México y los daños significativos causados por la plaga del pulgón amarillo, en 2015, el Servicio Nacional de Sanidad, Inocuidad y Calidad Agroalimentaria (SENASICA, 2014), a través de los Comités Estatales de Salud vegetal, autorizaron la campaña en contra del pulgón amarillo en sorgo. Esta campaña incluye acciones como el monitoreo, aplicación de control biológico, químico y cultural con énfasis en el entrenamiento para los productores.

En Nayarit, el Comité Estatal de Sanidad Vegetal (CESAVENAY) es responsable de la implementación de esta campaña. Las actividades llevadas a cabo por los técnicos del CESAVENAY durante estas inspecciones son las siguientes:

1. Se realiza la evaluación de la presencia de la plaga en el cultivo, si es el caso, se determina el nivel de infestación.

2. Se recolecta información relacionada con la parcela y el cultivo (fecha de siembra, etapa fenológica (ver Tabla 1), nivel de daño e información geográfica).

3. Se capacita a los productores de sorgo acerca del manejo del cultivo. Dicha capacitación se enfoca en la prevención y tratamiento de la plaga.

Los técnicos del CESAVENAY recolectan manualmente toda la información relacionada con el proceso de monitoreo de cultivos de sorgo en bitácoras. Después, capturan esta información en una plataforma web. Este proceso ha causado inconsistencias en la integridad de la información, principalmente en el registro de las coordenadas geográficas de la parcela evaluada, las etapas fenológicas del cultivo y los niveles de infestación reportados. Dichos problemas podrían ser atenuados si se automatiza el proceso de levantamiento de información haciendo uso de las nuevas tecnologías. 


\section{Etapa fenológica}

\begin{tabular}{lll}
\hline $\begin{array}{l}\text { Nombre según Vanderlip and Reeves } \\
(\mathbf{1 9 7 2})\end{array}$ & Nombre según CESAVENAY & No. de Días \\
\hline Siembra & Siembra & $\mathbf{1}$ \\
\hline Emergencia & Crecimiento & $\mathbf{2 0}$ \\
\hline Collar de tercera hoja visible & Desarrollo vegetativo & 30 \\
\hline Collar de quinta hoja visible & Elongación de tallo & 5 \\
\hline Diferenciación de punto de crecimiento & Floración & 6 \\
\hline Última hoja en espiral visible & Elongación de panícula & 8 \\
\hline Arranque de fruto & Formación del fruto & 10 \\
\hline Media floración & Desarrollo de panícula & 10 \\
\hline Masa blanda & Grano lechoso & 10 \\
\hline Masa dura & Grano masoso & 15 \\
\hline Madurez fisiológica & Madurez fisiológica & 25 \\
\hline Cosecha & Finalización del periodo de cosecha & 1 \\
\hline Total & & $\mathbf{1 4 1}$ \\
\hline
\end{tabular}

Tabal 1 - Etapas fenológicas del sorgo

Las nuevas tecnologías y paradigmas computacionales pueden ser empleados en diversos campos (Quezada-Sarmiento \& Mengual Andrés 2017), entre ellos la agricultura; el uso y manejo de dispositivos móviles constituyen una de las opciones que permiten agilizar el trabajo en diversas áreas (Razo Salas 2016).

Previamente, algunos investigadores han llevado a cabo esfuerzos de investigación relacionados con la automatización del proceso de monitoreo de cultivos y levantamiento de información. En algunos de estos trabajos se han desarrollado aplicaciones móviles; como el FarmBeats (Vasisht, y otros, 2017). Esta aplicación es parte de un sistema informático a través del cual el usuario puede visualizar los resultados del análisis de suelo y variables de salud en un cultivo. Este sistema contempla escenarios donde no hay conexión de Internet. El trabajo de Liopa-Tsakalidi, et al. (2013) describe un sistema que incluye diferentes herramientas (servicios web, una aplicación móvil basada en Android y una base de datos). Con esta aplicación móvil, y a través de sensores, el productor puede añadir información relacionada con el cultivo a una base de datos. Esta aplicación ayuda a tomar decisiones relacionadas con el desarrollo del cultivo.

En el presente trabajo proponemos una aplicación móvil llamada PulAm, la cual tiene como finalidad la automatización del monitoreo de diferentes cultivos, principalmente durante la captura de información de campo. Con esta aplicación, un técnico puede identificar parcelas, capturar automáticamente su información geográfica, y basado en su experiencia, determinar el nivel de infestación de un cultivo. Finalmente, como caso de estudio, proponemos el monitoreo de cultivos de sorgo afectados por la plaga del pulgón amarillo en ciertas regiones de Nayarit, México. Cabe mencionar que este trabajo es una extensión del trabajo presentado por Pérez-Mena et al. (2018) en el Congreso Internacional de Mejora de Procesos de Software 2018. 


\section{2. Área de estudio y proceso tradicional de monitoreo}

El área de estudio seleccionada es el estado mexicano de Nayarit, ver Figura 3, siendo éste el quinto productor de sorgo a nivel nacional. En Nayarit, el cultivo de sorgo es el segundo en orden de importancia en cuanto a la superficie sembrada. Anualmente se siembran más de 63,000.00 hectáreas de este grano en condiciones de humedad residual y riego. En agosto de 2014, la plaga del pulgón amarillo atacó por primera vez a los cultivos de sorgo en Nayarit, específicamente en el municipio de Amatlán de las cañas (CESAVENAY, 2015).

El Comité de Sanidad Vegetal de Nayarit (CESAVENAY) tiene un equipo de técnicos que se encargan del monitoreo de la plaga del pulgón amarillo en cultivos de sorgo. Planifican semanalmente jornadas de inspección en diferentes zonas cultivadas de sorgo. Hacen este diseño de acuerdo con los datos históricos de los cultivos inspeccionados, la presencia de la plaga y su grado de infestación.

En un día típico de inspección, el técnico llega en la mañana a su zona de inspección y distribuye control químico y/o biológico a los productores de sorgo de acuerdo con el nivel de infestación en su parcela. Después, los técnicos inspeccionan los cultivos de acuerdo con su planeación. Durante esta actividad, los técnicos registran nuevas parcelas si los productores lo requieren y evalúan el grado de infestación muestreando un cierto número de plantas en una parcela. Después registran en sus bitácoras la siguiente información: coordenadas del punto de inspección (obtenidas con un GPS), nombre de la plaga, nivel de infestación, número de plantas infestadas, etapa fenológica del cultivo inspeccionado, variedad de sorgo, fecha de inspección y otros datos ${ }^{1}$. Finalmente, el

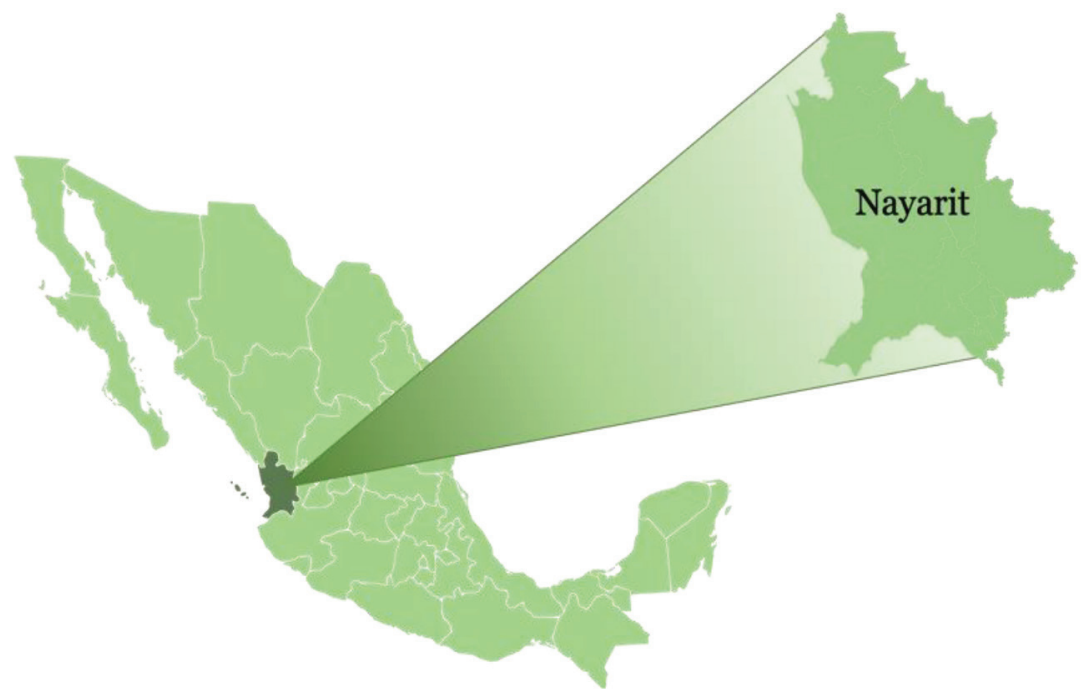

Figura 3 - Área de estudio

${ }^{1}$ Para realizar este estudio, personal del CESAVENAY proporcionó las bitácoras de la campaña del pulgón amarillo en Nayarit correspondientes a los años 2016 al 2018 
técnico ingresa manualmente la información obtenida durante su jornada a una plataforma Web propiedad del CESAVENAY.

Los problemas más frecuentes al realizar este proceso son los siguientes:

- Los técnicos pueden incurrir en errores durante el proceso de captura de la información de las inspecciones en sus bitácoras y después a la plataforma computacional donde almacenan esta información. Estos errores pueden ser de escritura o de inexactitud de datos.

- Los técnicos no siempre registran las nuevas coordenadas del punto deinspección, ya que éste a veces lo asocian a las coordenadas de un punto previamente obtenido de la parcela, no reflejando el punto exacto de la inspección.

- No hay forma de comprobar que los técnicos llevaron a cabo las inspecciones registradas en la bitácora.

- Los técnicos no tienen ninguna forma rápida para consultar el historial de información antes recabada.

- No hay suficientes técnicos para cuantificar los daños de todos los cultivos de sorgo.

\section{Propuesta}

En este trabajo, se introduce una aplicación móvil llamada PulAm para mitigar los problemas descritos en las secciones anteriores y para automatizar el proceso de monitoreo de los cultivos. Esta sección describe el objetivo de esta aplicación, su descripción técnica y sus componentes principales.

\subsection{Objetivos de PulAm}

El propósito principal de PulAm es dar soporte al personal técnico que se encarga de capturar toda la información relacionada con las inspecciones de campo, sin preocuparse de los detalles técnicos del proceso, como validaciones geográficas e integridad de los datos. PulAm tiene los siguientes objetivos particulares:

- Permitir la captura automática y confiable de la información de inspección.

- Mantener actualizada la información de las inspecciones para poder clasificar los daños causados por las plagas con información reciente. Esta actualización es automática cuando el dispositivo móvil, donde se encuentre instalada la aplicación, se conecte a Internet.

- Evitar la generación de errores al capturar información de la inspección.

- Consultar y editar la información relacionada con las inspecciones.

\subsection{Descripción técnica de PulAm}

PulAm se desarrolló para la versión de Android 4.0 (KitKat). El dispositivo móvil en el que PulAm se ejecute debe tener un GPS y conexión a Internet. La aplicación móvil necesita conexión a Internet porque debe conectarse a un servidor web para actualizar la información modificada, eliminada y agregada en la base de datos. En la Figura 4, se muestra la arquitectura de PulAm. Además de esta aplicación, el dispositivo Android hospeda una base de datos SQLite, de la que PulAm obtiene su información. El dispositivo 
se conecta al servidor a través de Internet y un servicio PHP para actualizar la información modificada o añadida a la base de datos MySQL descrita en la Sección 3.3.

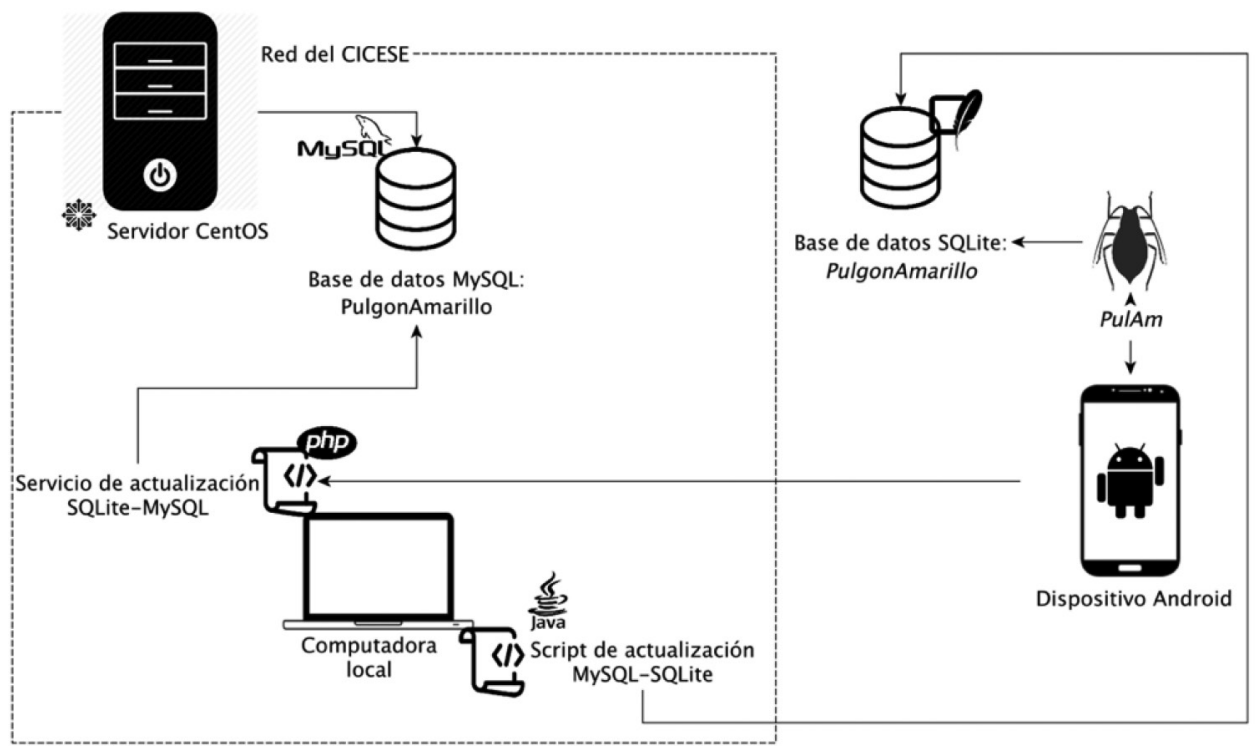

Figura 4 - Arquitectura de PulAm

Para el desarrollo de esta aplicación se utilizaron las siguientes librerías con su respectiva función en la aplicación:

- Librería Google maps. Se utiliza para desplegar los mapas de las capas de cultivos e inspecciones. Además, se usan funcionalidades de ubicación actual y obtención de coordenadas.

- Librería sqlite. Maneja las conexiones a la base de datos SQLite hospedada en el dispositivo Android.

- Librería volley. Maneja la conexión con el servicio web PHP para la sincronización de las bases de datos.

\subsection{Módulos de PulAm}

PulAm consiste de cinco módulos: (1) administración de la base de datos; (2) verificación de predios; (3) captura de la inspección; (4) personalización y (5) sincronización de la base de datos.

\subsubsection{Administración de la base de datos}

A partir de las bitácoras de la campaña del pulgón amarillo proporcionadas por el CESAVENAY, se extrae la información necesaria para crear la base de datos del proyecto, para lo cual se creó en el servidor una base de datos en MySQL, véase la Figura 4. 
Para la transmisión de esta información a la base de datos MySQL, se generó un programa en Java para agregar y validar de las inspecciones en las bitácoras. En la Figura 5, se pueden ver las tablas utilizadas en el proceso de almacenamiento de inspecciones. PulAm cuenta con una base de datos SQLite hospedada en el dispositivo móvil.

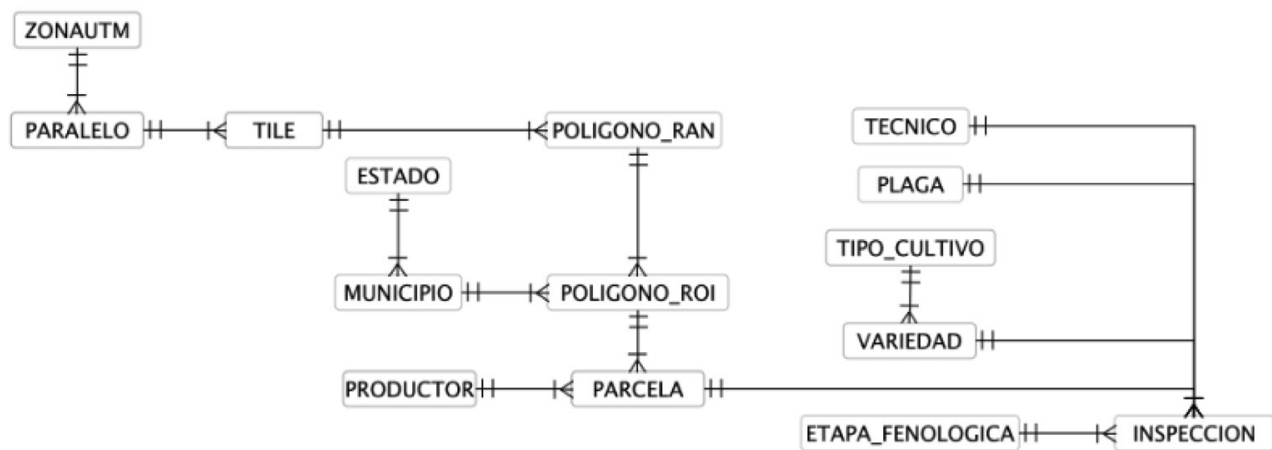

Figura 5 - Diagrama de la base de datos

En principio, los registros de inspección de las bitácoras no contienen información sobre la localización de las parcelas en donde se realizaron las inspecciones. Por lo que para obtener su información geográfica se siguieron los siguientes pasos:

1. Se obtuvo la información geográfica de las regiones agrarias de Nayarit a través del Registro Agrario Nacional (polígonos RAN). Las regiones agrarias son zonas de cultivos de especies vegetales.

2. Se obtuvieron las coordenadas de inspección en formato shapefile de puntos.

3. Se conjuntaron la capa de coordenadas y la capa de polígonos RAN para obtener únicamente los polígonos que contienen inspecciones.

4. Se hizo la sobreposición de la imagen del polígono RAN con la capa de coordenadas de inspecciones con la ayuda del software QGIS.

5. Por cada inspección, se dibujó el polígono de la región de interés de la parcela o polígono ROI, guardándose en un shapefile.

El módulo de manejo de la base de datos es responsable de la consulta, edición, eliminación y adición de información en las siguientes tablas de la base de datos: inspección, plaga, tipo de cultivo, productor, técnico y cultivos (ver Figura 6 (a)-(f)). El manejo de cada tabla tiene restricciones relacionadas con la prioridad de la información y cómo la modificación afecta a otras tablas.

Cabe destacar que el técnico no puede agregar inspecciones desde esta sección, sólo las puede consultar y editar. Con el fin de minimizar problemas de conectividad, se implementó una base de datos local en el dispositivo móvil en lugar de un servicio web; lo cual permite que la aplicación funcione incluso donde no hay señal de Internet. 
(a)

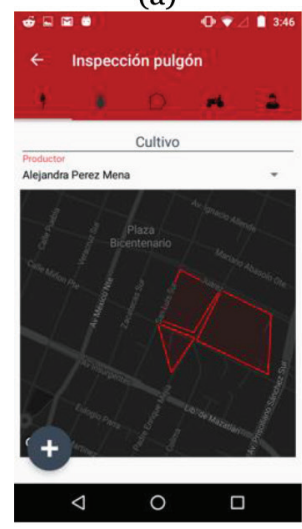

(e)

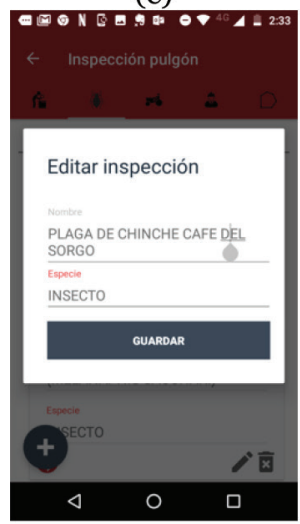

(b)

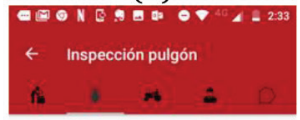

Plaga

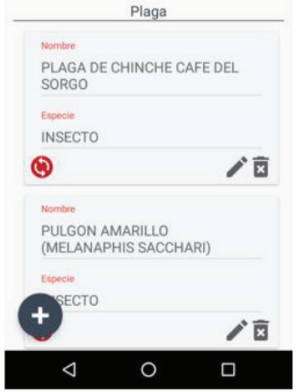

(f)

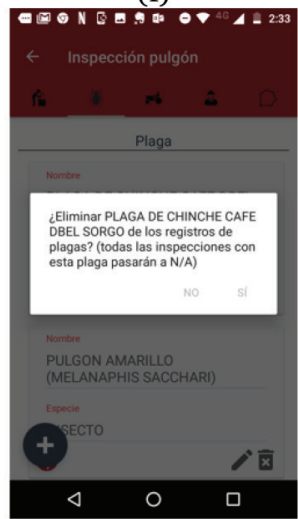

(c)

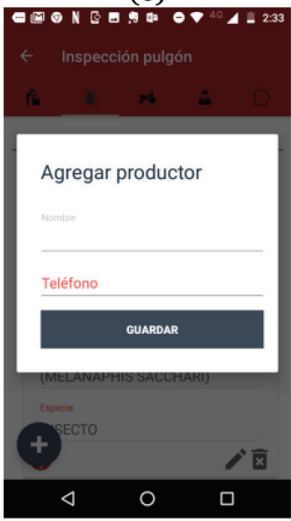

(g)

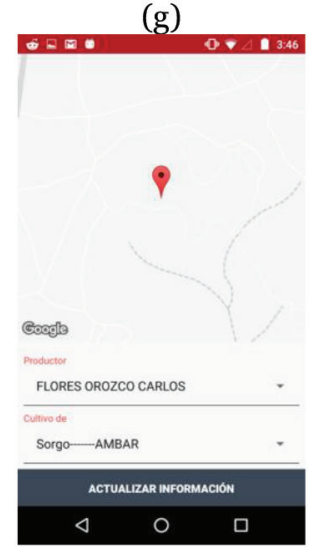

(d)

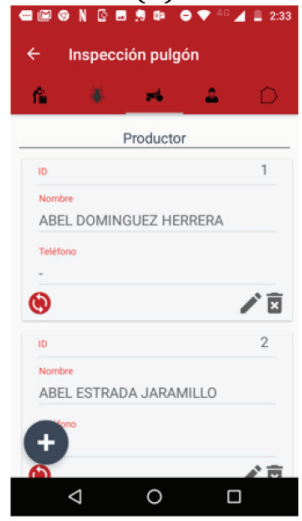

$\square$

Figura 6 - Módulo de administración de base de datos y verificación de predio

\subsubsection{Verificación de cultivos}

En el módulo de verificación de cultivos, el usuario puede seleccionar a quién le pertenece una parcela. Además, muestra quién se encuentra actualmente trabajando en la parcela y qué tipo de cultivo está sembrado. Se implementó este módulo debido a que existen predios cuyo productor actual no corresponde al registrado en la base de datos (ver Figura $6(\mathrm{~g})$ ).

\subsubsection{Captura de la inspección}

En el módulo de captura de inspección (ver Figura 7), el técnico puede capturar el nivel de infestación de la plaga en el cultivo en el que se encuentre en ese instante. PulAm automáticamente captura las coordenadas, fecha y hora de la inspección, y completa el registro de inspección con la información de la última inspección añadida. El técnico 
puede modificar la información de la inspección que está por añadir, con excepción de las coordenadas, fecha y hora de la inspección.

El módulo de captura de la inspección consiste de tres pantallas: (a) el mapa (donde se visualiza la ubicación del técnico en el cultivo); (b) la adición rápida de la inspección (donde si el usuario ingresa el nivel de infestación y da clic en Guardar, la aplicación almacena la información restante de la última inspección añadida); y (c) la pantalla donde el técnico puede modificar la información de la inspección capturada. En caso de que el usuario esté ubicado en un punto donde no exista un cultivo registrado en la base de datos, debe crear un nuevo cultivo para agregar una inspección. Esto se hace por medio de la sección de administración de la base de datos.

(a)

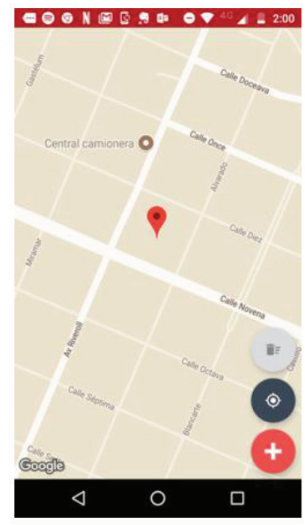

(b)

Agregar inspección

Nivel de infestación \%

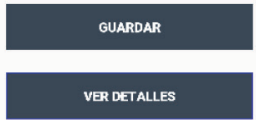

(c)

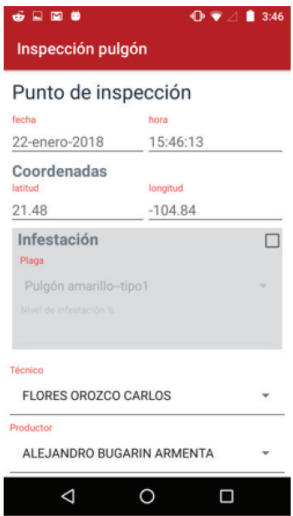

Figura 7 - Módulo captura de inspección

\subsubsection{Personalización de PulAM}

PulAm permite a los usuarios personalizar las siguientes características: tipo de mapa usado (por defecto el mapa cambia de color de acuerdo con el momento del día) y el técnico que usa la aplicación (ver Figura 8).

\subsubsection{Sincronización de la base de datos}

El módulo de sincronización de la base de datos es responsable de la sincronización de todos los registros eliminados, editados y añadidos por medio de PulAm cuando el dispositivo se conecta a Internet. Cada vez que un usuario cambia algo en la base de datos, la consulta entra a una cola de consultas. Una vez que el dispositivo se conecta a Internet, este módulo manda la información al servidor mediante el protocolo HTTP que captura las consultas con un servicio PHP.

Finalmente, el servidor ejecuta las consultas en la base de datos MySQL. Para la conversión de la base de datos MySQL a SQLite se pueden utilizar distintos programas de conversión; sin embargo, éstos requieren supervisión o tienen un costo por uso, por lo que se generó un programa en Java que convierte la base de datos de MySQL a SQLite antes de la generación en producción de la aplicación. Además, este módulo agrega nuevos 
(a)

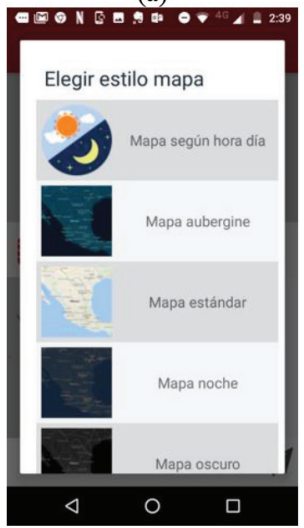

(b)

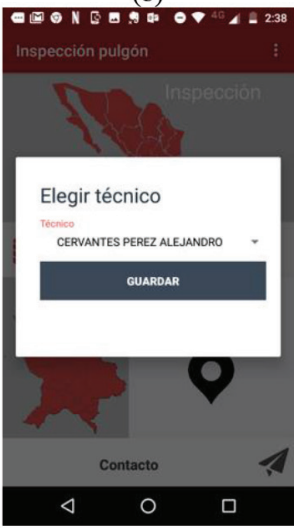

Figura 8 - Módulo de personalización

datos de clasificación en la base de datos. No se implementó una base de datos MySQL en el dispositivo móvil debido a que demanda muchos recursos computacionales en comparación con una base de datos SQLite. Este módulo es útil cuando no hay conexión a Internet disponible; la información permanece en la cola hasta que se puede sincronizar.

\section{Inspecciones a cultivos de Nayarit y pruebas funcionales de la aplicación}

Para probar PulAm, llevamos a cabo una campaña de monitoreo, la cual consistió en visitar 40 plantaciones de sorgo. Para realizar esta actividad se solicitó al CESAVENAY copia de las bitácoras de las inspecciones a cultivos de sorgo y que el primer autor de este

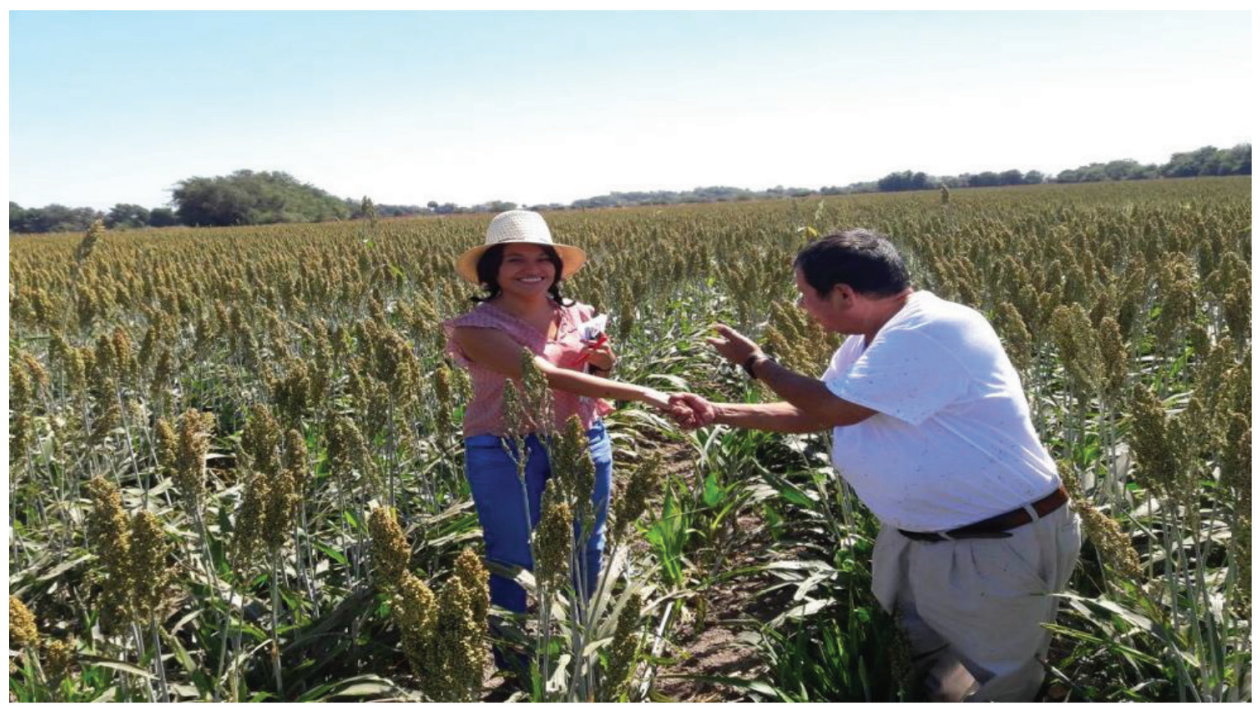

Figura 9 - Campaña de monitoreo de campos de sorgo 
trabajo pudiera estar presente en las campañas fitosanitarias del pulgón amarillo, ver Figura 9. Las inspecciones se llevaron a cabo en los municipios de nayaritas de Rosamorada, Santiago Ixcuintla y Tuxpan.

Durante la fase de inspección a cultivos, se recibió capacitación para reconocer el grado de infestación en el cultivo y en las plantas. Para evaluar el daño, se eligen plantas al azar y por planta se hace un estimado del número de pulgones que existen en ella. Como resultado, se obtuvo la información de cultivos de sorgo en cinco de sus etapas fenológicas: madurez fisiológica, cosecha, grano lechoso, grano masoso y panoja cerrada.

\section{Resultados}

Se obtuvo como producto una base de datos llamada pulgonamarillo la cual almacena información histórica de inspecciones (8411 inspecciones). En la Tabla 2, se muestra el total de inspecciones por año.

\begin{tabular}{lc}
\hline Año & Inspecciones \\
\hline 2016 & 3717 \\
\hline 2017 & 4085 \\
\hline 2018 & 610 \\
\hline Total & $\mathbf{8 4 1 2}$ \\
\hline
\end{tabular}

Tabal 2 - Inspecciones

En la Figura 10, se observa la cantidad de inspecciones por etapa fenológica que existe en la base de datos. La etapa fenológica con más inspecciones registradas es la de cosecha,

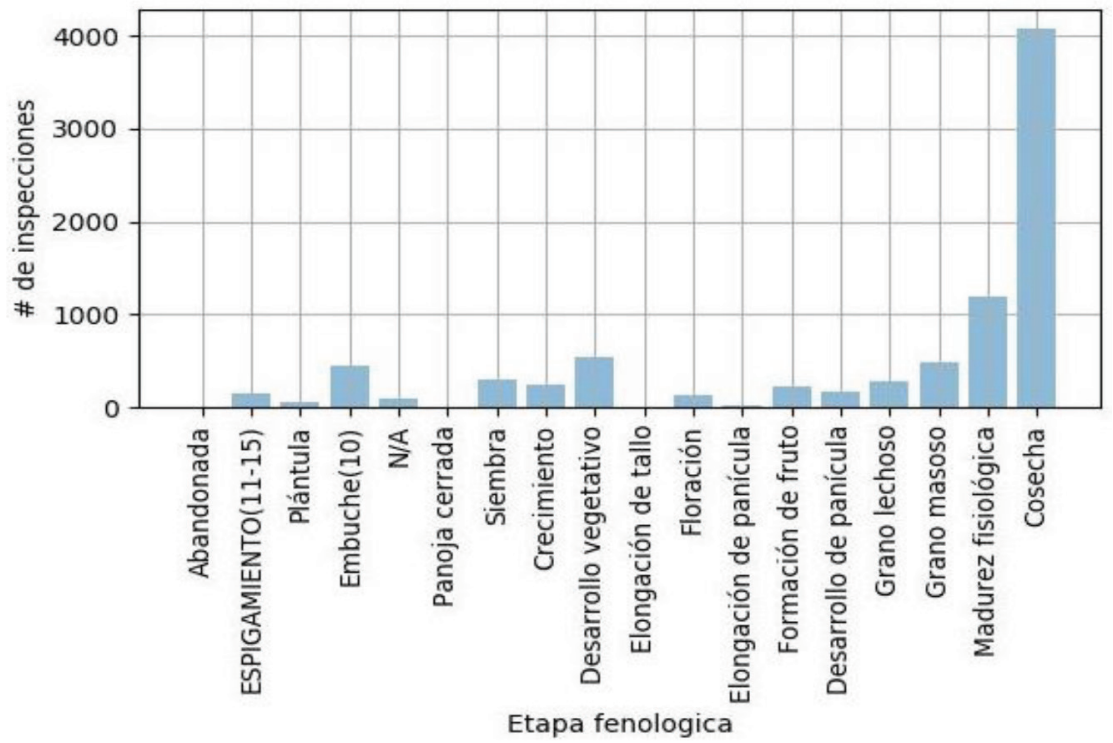

Figura 10 - Inspecciones por etapa fenológica 
seguida por la de madurez fisiológica. Esto se debe a que los técnicos del CESAVENAY marcan como cosecha toda aquella parcela en la que no hay nada cultivado.

En la Figura 11, se muestran los histogramas de las inspecciones por nivel de infestación obtenidas durante el llenado de la base de datos. En estos histogramas se puede ver cuál es la distribución de los niveles de infestación en las diferentes etapas fenológicas del cultivo del sorgo.

(a) Abandonada

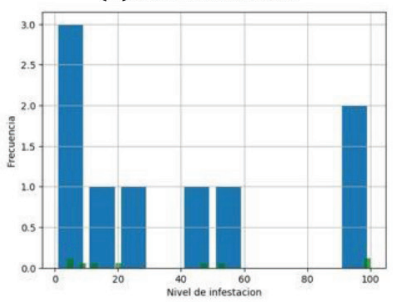

(d) Embuche

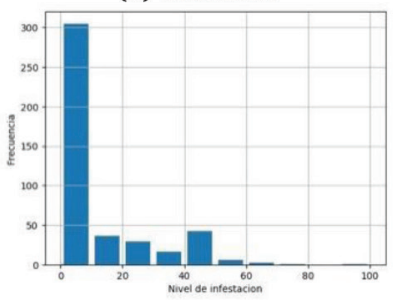

(g) Siembra

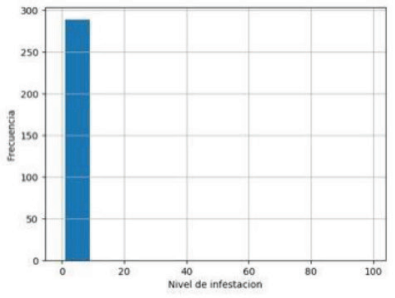

(j) Elongación del tallo

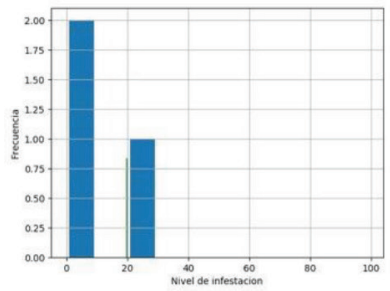

(b) Espigamiento

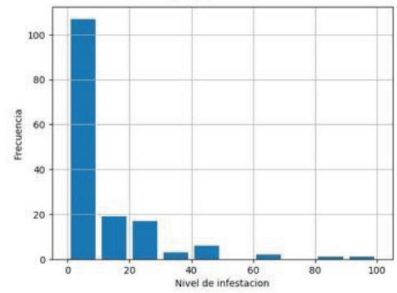

(e) Ninguna

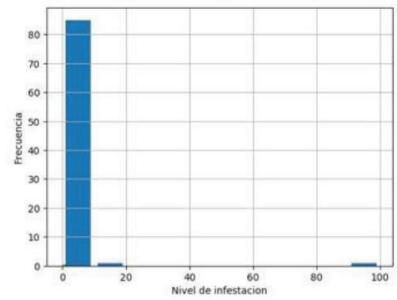

(h) Crecimiento

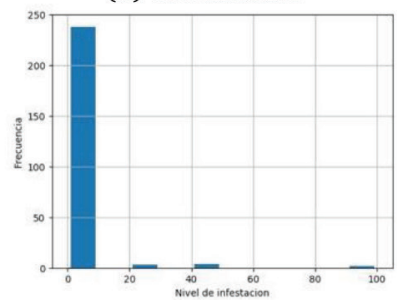

(k) Floración

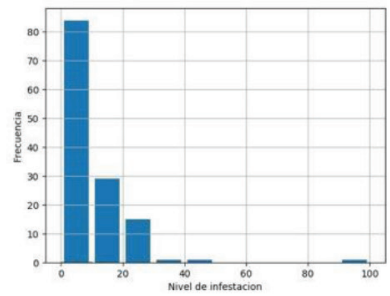

(c) Plántula

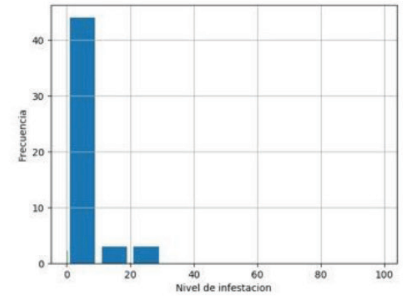

(f) Panoja cerrada

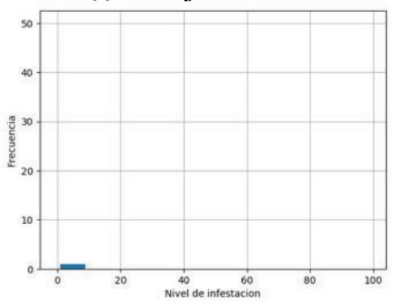

(i) Desarrollo vegetativo

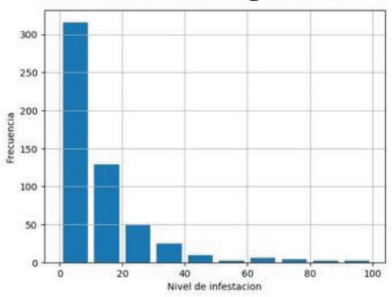

(1) Elongación de la panícula

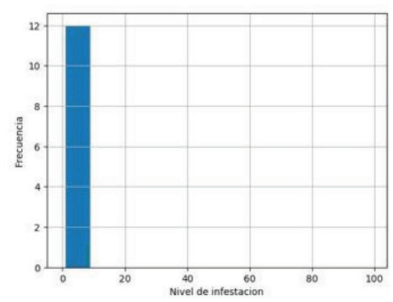

Figura 11 - Histogramas por etapa fenológica 
Del análisis de estos datos se obtiene lo siguiente:

- En la mayoría de las inspecciones, el nivel de infestación es o\%, excepto en la etapa fenológica de formación de fruto, donde la mayoría de las inspecciones reportan niveles del 20 al $30 \%$.

- Las inspecciones con una distribución más variada de niveles de infestación son las de abandonada, embuche, desarrollo vegetativo y formación de fruto.

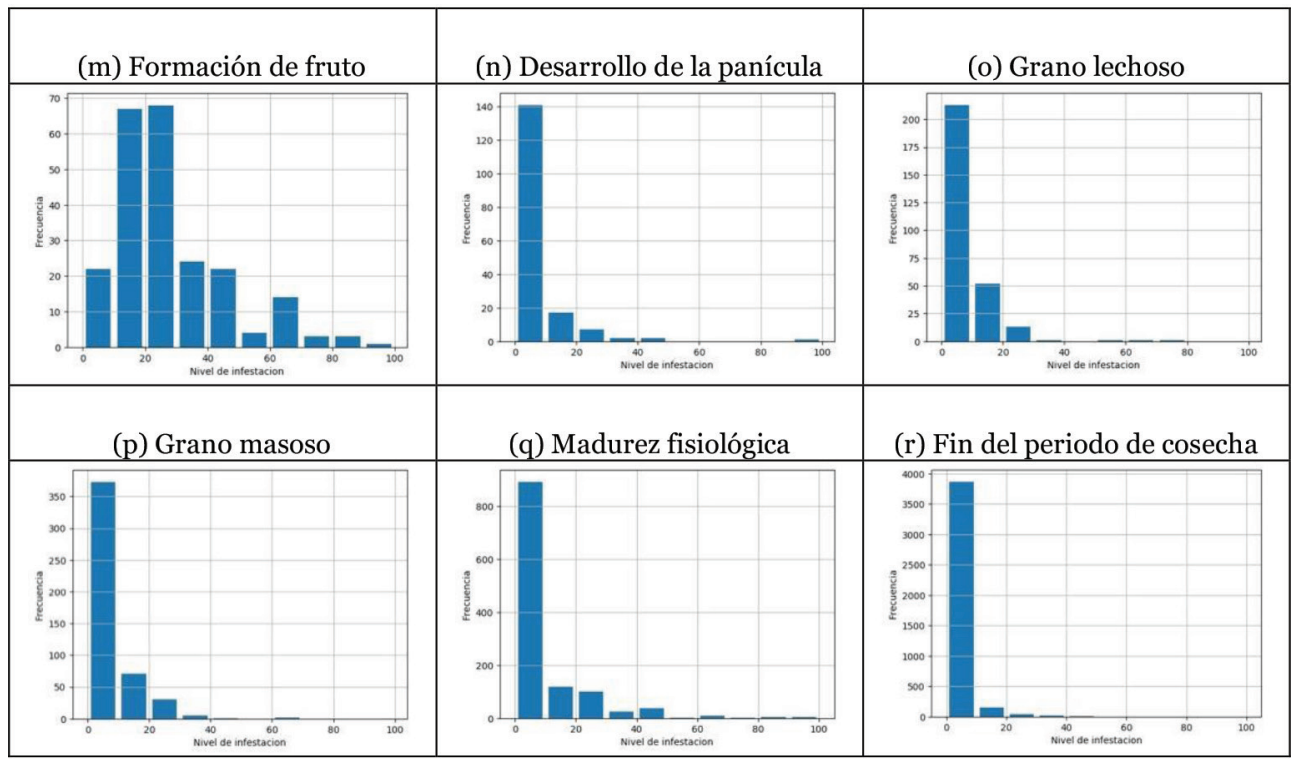

Figura 12 - Histogramas por etapa fenológica (continuación)

Con la información recopilada, se identificaron algunas mejoras necesarias para la aplicación. Posteriormente, se realizaron pruebas de funcionalidad a los cuatro módulos principales de PulAm. A partir de estas pruebas, se pudieron realizar las siguientes mejoras:

- El usuario tuvo que seleccionar su nombre manualmente cada vez que quería agregar nuevas inspecciones. Posteriormente, se agregó una opción en el menú de preferencias, por lo que el usuario debe hacerlo sólo una vez.

- Se configuró el dispositivo móvil de tal manera que, en ausencia de una señal GPS, el dispositivo puede solicitar los datos más precisos disponibles a través de Wi-Fi y las torres celulares del proveedor de la red.

- Se agregó un cuadro de diálogo en el módulo de captura de inspección para facilitar la adición de una nueva inspección. Ahora, el usuario sólo debe agregar el grado de infestación mientras la aplicación toma el resto de la información de la última inspección agregada, información que se almacena automáticamente en el archivo de preferencias compartidas. Finalmente, el usuario puede editar esta información de acuerdo con los datos de la inspección actual. 
- PulAm inicialmente usó una serie de puntos almacenados en la base de datos que juntos formaron un polígono representado por una plantación. La aplicación tuvo que dibujar todos los puntos para formar el polígono, usando recursos computacionales altos. Ahora, PulAm lee los polígonos como una capa geográfica que dibuja en el mapa rápidamente.

- Se añadieron funciones adicionales en el módulo de gestión de bases de datos.

\section{Conclusiones}

PulAm es una aplicación móvil basada en el sistema operativo Android, la cual es muy útil para el levantamiento de datos en campo. Como se mostró en el presente trabajo de investigación, PulAm es un prototipo a través del cual los técnicos de CESAVENAY pueden registrar el levantamiento de datos de sus campañas fitosanitarias, de forma ágil y minimizando los errores. Además, pueden acceder a información histórica sobre inspecciones realizadas previamente.

Como trabajo futuro, se planea realizar pruebas de aceptación de PulAm con el personal técnico de CESAVENAY. También se planea diseñar un módulo en el que el usuario pueda tomar una o más fotografías de las plantas de algún cultivo con su dispositivo móvil y que la aplicación pueda determinar automáticamente el grado de infestación. Con este módulo, el usuario tendría una medición más estandarizada del nivel de infestación.

Agradecimientos. Agradecemos al personal de CESAVENAY por toda su ayuda, tanto técnica como logística para la recolección de datos en el campo. Alejandra Perez-Mena agradece al Consejo Nacional de Ciencia y Tecnología (CONACYT) por la subvención financiera otorgada a través de la beca \#613382.

\section{Referencias}

CESAVENAY. (2015). Campaña contra el pulgón amarillo. México: Comité Estatal de Sanidad Vegetal de Nayarit.

Liopa-Tsakalidi, A., Tsolis, D., Barouchas, P., Chantzi, A.-E., Koulopoulos, A., \& Malamos, N. (2013). Application of Mobile Technologies through an Integrated Management System for Agricultural Production. Procedia Technology, 8, 165-170.

INEGI. (2016). Actualización del Marco Censal Agropecuario 2016. Obtenido de https://www.inegi.org.mx/proyectos/agro/amca/

INEGI. (2019). Mapa Digital de México. Obtenido de http://gaia.inegi.org.mx/mdm6/

Montoya, F. G., Gómez, J., Cama, A., Zapata-Sierra, A., Martínez, F., De La Cruz, J., \& Manzano-Agugliaro, F. (2013). A monitoring system for intensive agriculture based on mesh networks and the android system. Computers and Electronics in Agriculture, 99, 14-20. 
Pérez-Mena, A., Fernández-Zepeda, J. A., Rivera-Caicedo, J. P., \& Avila-George, H. (2019). PulAm: An App for Monitoring Crops. En: J. Mejia, M. Muñoz, Á. P. Rocha, \& M. Pérez-Cisneros (Eds.), Proceedings of the 7 th International Conference on Software Process Improvement (CIMPS 2018) (Vol. 865, págs. 196-205). Guadalajara, México: Springer.

Quezada-Sarmiento, P. A. \& Mengual Andrés, S. (2017). Implementación de una solución web y móvil para la gestión vehicular basada en Arquitectura de Aspectos y metodologías ágiles: Un enfoque educativo de la teoría a la práctica. RISTI-Revista Ibérica de Sistemas y Tecnologías de Información, 27, 97-111.

Razo Salas, M. A., Vázquez Reyes, S. \& Solís Robles, R. (2016). Mejoras del proceso endoscópico usando aplicaciones móviles. RISTI-Revista Ibérica de Sistemas y Tecnologías de Información, 17, 115-127.

Rodríguez-del-Bosque, L. A., \& Terán, A. P. (2015). Melanaphis sacchari (Hemiptera: Aphididae): A New Sorghum Insect Pest in Mexico. Southwestern Entomologist, 40(2), 433-434.

SAGARPA. (2014). Atlas agroalimentario México. México: Secretaría de Agricultura, Ganadería, Desarrollo Rural, Pesca y Alimentación.

SENASICA. (2014). Pulgón amarillo Melanaphis sacchari (Zehntner). México: Servicio Nacional de Sanidad, Inocuidad y Calidad Agroalimentaria.

SIAP. (2017). Servicio de Información Agroalimentaria y Pesquera. Obtenido de: http://infosiap.siap.gob.mx

Tezer, O. S. (2014). SQLite vs MySQL vs PostgreSQL: A Comparison Of Relational Database Management Systems. Obtenido de: https://www.digitalocean.com/ community/tutorials/sqlite-vs-mysql-vs-postgresql-a-comparison-of-relationaldatabase-management-systems

Vanderlip, R. L., \& Reeves, H. E. (1972). Growth Stages of Sorghum (Sorghum bicolor, (L.) Moench.). Agronomy journal, 64(1), 13-16.

Vasisht, D., Kapetanovic, Z., Won, J.-h., Jin, X., Chandra, R., Kapoor, A., . . Stratman, S. (2017). Farmbeats: an IoT platform for data-driven agriculture. En: A. Akella, \& J. Howell (Ed.), Proceedings of the 14th USENIX Symposium on Networked Systems Design and Implementation (págs. 515-529). Boston, MA, USA: USENIX Association. 
